\title{
Adaptive control scheme for maximum power point tracking of a photovoltaic system connected to the grid.
}

\author{
Fernando Jaramillo-Lopez, Gilney Damm, Godpromesse Kenne and Francoise Lamnabhi-Lagarrigue
}

\begin{abstract}
An adaptive control scheme for maximum power point tracking of a single-phase single-stage photovoltaic system connected to the grid is presented.

The maximum power point depends on temperature and solar irradiance, ambient conditions that are time-varying and difficult to measure.

Two solutions are presented. Each solution derive an estimator that approximate three different parameters. These parameters are functions of solar irradiance and temperature. In this manner, we eliminate the necessity of climatic sensors. The first solution, uses an adaptive estimator that is able to estimate constant parameters, and the second one uses a sliding mode estimator that is capable of estimate time-varying parameters.

A complete analysis was done taking into account the nonlinearities showed by the closed-loop system. The Lyapunov redesign technique was used to derive a controller that gives globally asymptotically stable trajectories of the closed-loop system. Computer simulations are presented to compare the performance of both estimators and also to show the good performance of the controller.
\end{abstract}

\section{INTRODUCTION.}

New policies and regulations have been developed to face the growing energy needs and climate change. These facts have stimulated the interest on renewable energy sources. Solar photovoltaic is one of them. Photovoltaic systems (PVS) converts sunlight directly into electricity by means of a semiconductive process.

Grid-connected PVS usually consists of a photovoltaic panel or array and a power conditioning system (PCS).

In [1] and [2], the authors analyze advantages and disadvantages of various single-phase grid-connected inverters topologies. The single-phase single-stage central inverter DC/AC topology was chosen in this work, because of its simplicity and low price.

The output power of a photovoltaic array is function of irradiance and temperature. To increase the efficiency of the overall system, PVS always needs to work in its maximum power point, to deliver the maximum amount of energy (Fig. 1). Hence, an algorithm that can follow these power changes is needed. This is the maximum power point tracking (MPPT) algorithm.

\footnotetext{
* This research has received partially support from European Union Seventh Framework Programme [FP7/2007-2013] under grant agreement no.257462 HYCON2 Network of excellence. *

F. Lamnhabi and F. Jaramillo are with the Laboratoire des Signaux et Systèmes, Supelec, Plateau du Moulon, 91192 Gif-sur-Yvette, France. lamnhabi jaramillo\}alss.supelec.fr

G. Damm is with IBISC, Université Evry, Val d'Essonne, France. gilney.dammeibisc.fr

G. Kenne is with LAIA, Département de Genie Electrique, IUT-FV Bandjoun Université de Dschang, Cameroun. gokenne@yahoo. com
}

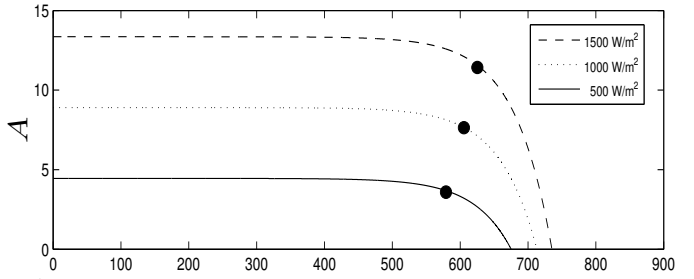

a) voltage vs current curve at different irradiance levels

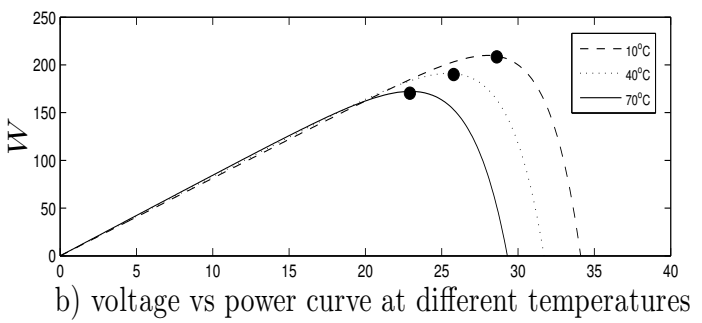

Fig. 1. MPP of a typical PV array for different environmental conditions a) Voltage vs. current curve at $T=25^{\circ} \mathrm{C}$ and different irradiance values b) Voltage vs. power curve at $G=1000 \mathrm{~W} / \mathrm{m}^{2}$ and different temp. values.

There are several MPPT algorithms like perturb and observe, incremental conductance, extremum-seeking among others. They compute the value of the voltage corresponding to the maximum power point. This voltage is then used as a reference value in the controller. In [3] and [4] the authors present some improvements and comparisons between these algorithms.

Previous works on MPPT control of PVS were done by splitting the problem in two parts: a control capable of tracking the MPP and a control capable of deliver a sinusoidal current in phase with the grid voltage.

Solutions like feedback linearization and sliding mode techniques were applied to the first part.

Several solutions have been developed for the second part, like the $\mathrm{P}+$ resonant controller [5] and the adaptive resonant controller [6]. However these solutions are linear based techniques. Approximations that doesn't take into account the nonlinear behavior of the PV array.

An interesting solution that uses the nonlinear model of PVS was presented in [7]. In this work, the MPPT and the unity power factor tasks are satisfied with a single passive control, based on the work of [8]. MPP tracking is accomplished for time-varying environmental conditions, with the help of an estimator that compute a reference for the controller. However, global stability with the use of this 
estimator is not proved.

In [9] an attempt to prove global stability for the whole system was made. In this case, an estimation of the parameter that depends on irradiance and temperature is used together with the Lyapunov-based controller to prove global stability. However, in that work it is assumed that another two parameters that depends on temperature are known.

In this paper, we are presenting two solutions that uses an adaptive scheme control that is capable of achieving MPP tracking for changing environmental conditions and deliver unity power factor current to the grid. In the first solution, an adaptive estimator is designed, and in the second one, a sliding mode estimator capable of estimate time-varying parameters. Both, estimate parameters that depends on irradiance and temperature. No assumption about known parameters was made and global stability is proved in both cases, taking into account the nonlinear model and the estimates of the parameters. The main contribution consists in the full analysis - nonlinear model, controller and estimators- of the system, increasing of the robustness and elimination of the necessity of irradiance and temperature sensors with these estimators.

\section{PROBLEM FORMULATION AND MATHEMATICAL MODEL OF THE PHOTOVOLTAIC SYSTEM.}

The system analyzed in this paper is shown in figure 2 and consists in a PV array of solar cells and a DC/AC PCS. The states of the system are given by the capacitor voltage $\left(x_{1}\right)$ and the inductor current $\left(x_{2}\right)$. The PCS is a full-bridge inverter driven by a bipolar PWM scheme. The PWM gives two discrete complementary signals $s$ and $\bar{s}$ which turn on and off the four switches in the PCS. The PWM block is fed by the control signal $u \in[-1,1]$.

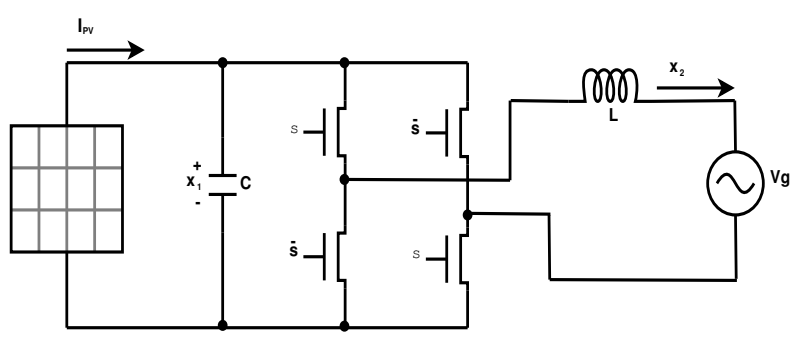

Fig. 2. Photovoltaic system analyzed in this paper.

\section{A. Mathematical model.}

The PV array is composed by PV cells arranged in series and parallel. The PV cell model used in this work is the single-diode model with no resistors. In [10] and [11], the authors present some models and give explicit relations to get the electric characteristics which are functions of irradiance and temperature. A comparative analysis was made in [12], and it is shown that all models have no significant differences for MPPT purposes.
The $I-V$ characteristic curve of a PV array with identical cells is given by:

$$
I=I_{p h}(G) N_{p}-I_{o}(T) N_{p}\left[\exp \left(\frac{q V}{n N_{s} k_{B} T}\right)-1\right]
$$

where $I_{p h}$ is the photocurrent, that depends on irradiance $G$, $I_{O}$ is the saturation current, that depends on temperature $T$, $q$ is the absolute value of electron's charge, $n$ is the quality factor of the diode, $N_{p}$ is the number of cells connected in parallel, $N_{s}$ is the number of cells connected in series, $k_{B}$ is Boltzmann's constant and $T$ is the temperature of the P-N junction. $V$ is the capacitor voltage $x_{1}$.

This equation can be written in a simpler way:

$$
I\left(x_{1}\right)=c_{1}-c_{2} e^{c_{3} x_{1}}
$$

where $c_{1}$ is function of irradiance and temperature and $c_{2}$ and $c_{3}$ are functions of temperature.

The model of the whole system is given by:

$$
\dot{x}=f(t, x)+G(t, x) u
$$

where

$$
f(t, x) \triangleq\left[\begin{array}{c}
\frac{I\left(x_{1}\right)}{C} \\
\frac{-v_{g}(t)}{L}
\end{array}\right], G(t, x) \triangleq\left[\begin{array}{c}
\frac{-x_{2}}{C} \\
\frac{x_{1}}{L}
\end{array}\right]
$$

$C$ and $L$ are known values of the capacitor and the inductor respectively, and $v_{g}$ the grid voltage. The signals that are measured are the states $x \in \mathbb{R}^{2}$, the voltage grid $v_{g} \in \mathbb{R}$ and the PV array current $I \in \mathbb{R}$, which is a common practice in this type of circuits.

$u$ is the control signal composed by the terms:

$$
u=u_{n}+w+\delta(t, x)
$$

$\delta(t, x)$ is an uncertain term that satisfies the matching condition (i.e. it enters at the same point that the control signal $u$ ), $w$ is the term that will be derived to compensate it and $u_{n}$ is the control component that turn the nominal system globally asymptotically stable. In section IV, these components are explained in detail.

The nominal system is the system without the uncertain term $\delta(t, x)$, hence:

$$
\dot{x}=f(t, x)+G(t, x) u_{n}
$$

where $f(t, x)$ and $G(t, x)$ are defined in the same way that before.

The main tasks that the signal control $u$ must fulfill are:

1) To track the maximum power point of the PV array, despite of changes in the environmental variables irradiance and temperature.

2) To deliver a current in phase with the grid voltage (i.e. unity power factor).

The controller needs two references to accomplish the main tasks of the system. The first reference is the voltage DC value in the capacitor $\bar{x}_{1 *} \in \mathbb{R}_{+}$and is given by the 
MPPT algorithm. The second reference is the current value in the inductor $x_{2 *}$, which is taken from [9]:

$$
x_{2 *} \triangleq \frac{2 v_{g}(t) \bar{x}_{1 *} I_{*}\left(\bar{x}_{1 *}, c_{i}\right)}{A^{2}}
$$

where $I_{*}$ is the PV current (2) evaluated at $\bar{x}_{1 *}$ and $v_{g}$ is assumed to be sinusoidal with constant amplitude $A$ and frequency $\omega, v_{g}=A \sin (\omega t)$.

The parameters $c_{i}$ in $I_{*}$ are unknown. Therefore, two different estimators were designed and presented here. They allow to calculate the full $I_{*}$ expression.

Hence, the second reference becomes:

$$
\hat{x}_{2 *} \triangleq \frac{2 v_{g}(t) \bar{x}_{1 *} \hat{I}_{*}\left(\bar{x}_{1 *}, \hat{c}_{i}\right)}{A^{2}}
$$

where $(\hat{\cdot})$ indicate the estimate of $(\cdot)$.

\section{ESTIMATORS DESIGN}

A reparametrization was made in order to be able to design the next two estimators.

From (2) and (6) the derivative of the current $I$ is:

$$
\begin{aligned}
\dot{I} & =-c_{2} c_{3} \dot{x_{1}} e^{c_{3} x_{1}} \\
& =\frac{1}{C}\left(u x_{2}-I\right)\left(\theta_{1}-\theta_{2} I\right)=\Phi^{T} \theta
\end{aligned}
$$

where:

$$
\theta \triangleq\left[\begin{array}{c}
c_{1} c_{3} \\
c_{3}
\end{array}\right], \Phi^{T} \triangleq \frac{1}{C}\left[\begin{array}{ll}
u x_{2}-I, & -u x_{2} I+I^{2}
\end{array}\right]
$$

also consider the following estimator and observer errors respectively, $\tilde{\theta}=\hat{\theta}-\theta, \tilde{I}=\hat{I}-I$ for the next subsections.

\section{A. Adaptive estimator.}

An adaptive estimator [13] capable of estimate constant parameters is designed in this subsection.

The estimator is given by:

$$
\begin{gathered}
\dot{\hat{I}}=-\lambda(\hat{I}-I)+\Phi^{T} \hat{\theta} \\
\dot{\hat{\theta}}=-\Gamma \Phi(\hat{I}-I)
\end{gathered}
$$

where $\lambda>0$ and $\Gamma$ is a square diagonal matrix $2 \times 2$ with positive values.

Let us assume the following:

Assumption 1-A. The parameters vector $\theta$ is constant, i.e.

$$
\dot{\theta}=0
$$

Lemma 1. Consider the observer (11) and the estimator (12), satisfying assumption $1-\mathrm{A}$, then the product $\Phi^{T} \tilde{\theta}$ converges to zero.

Proof. Let's first rewrite the estimation errors as:

$$
\begin{gathered}
\dot{\tilde{I}}=-\lambda \tilde{I}+\Phi^{T} \tilde{\theta} \\
\dot{\tilde{\theta}}=-\Gamma \Phi \tilde{I}
\end{gathered}
$$

Consider the following Lyapunov candidate function

$$
V=\frac{1}{2}|\tilde{I}|^{2}+\frac{1}{2} \tilde{\theta}^{T} \Gamma^{-1} \tilde{\theta} \triangleq \beta(\tilde{I}, \tilde{\theta})
$$

Its time derivate is

$$
\dot{V}=-\lambda|\tilde{I}|^{2}+\tilde{I}^{T} \Phi^{T} \tilde{\theta}-\tilde{\theta}^{T} \Phi \tilde{I}=-\lambda|\tilde{I}|^{2}
$$

By Barbalat's lemma, we can conclude that:

$$
\tilde{I} \rightarrow 0 \text { as } t \rightarrow \infty
$$

Therefore, it is clear by (13) that $\Phi^{T} \tilde{\theta} \rightarrow 0$ as $t \rightarrow \infty$

Furthermore, if the regressor $\Phi(t)$ satisfies the persistent excitation condition [14]:

$$
\int_{t}^{t+T_{0}} \Phi(\tau) \Phi^{T}(\tau) d \tau \geq \alpha I_{m} \quad \forall t \geq t_{0}
$$

where $I_{m}$ is the identity matrix and $t_{0}, T_{0}$ and $\alpha$ are positive constants, then we can in addition conclude that

$$
\tilde{\theta} \rightarrow 0 \text { as } t \rightarrow \infty
$$

\section{B. Sliding mode estimator.}

In [15] a new framework for nonlinear systems timevarying parameter estimation using sliding mode techniques was proposed.

The following notation is used in this subsection:

$$
\left|x^{T}\right|_{G}=\left(\left|x_{1}\right|,\left|x_{2}\right|, \ldots\left|x_{n}\right|\right)
$$

$\operatorname{diag}(A)$ is the column vector whose elements are the diagonal elements of a given square diagonal matrix $A$.

The function $\operatorname{sign}(\cdot): \mathbb{R}^{n} \rightarrow \mathbb{R}^{n}$ is defined as

$$
\operatorname{sign}\left(x^{T}\right)=\left(\operatorname{sign}\left(x_{1}\right), \operatorname{sign}\left(x_{2}\right), \ldots \operatorname{sign}\left(x_{n}\right)\right)
$$

with

$$
x^{T}=\left(x_{1}, x_{2}, \ldots x_{n}\right)
$$

and

$$
\operatorname{sign}\left(x_{i}\right)\left\{\begin{array}{lll}
=-1 & \text { for } & x_{i}<0 \\
=[-1,1] & \text { for } & x_{i}=0 \\
=1 & \text { for } & x_{i}>0
\end{array}\right.
$$

Now, let us assume the following:

Assumption 1-B. The derivatives of the parameters vector $\theta$ are bounded and these bounds are known:

$$
\dot{\theta}_{i}(t) \leq \mu_{\theta_{i}}
$$

where $\mu_{\theta i}$ are known positive numbers and $i=1,2$.

In order to design the time-varying parameter estimator consider the following adaptive observer for the current $I$ :

$$
\dot{\hat{I}}=v_{I}+\Phi^{T} \hat{\theta}
$$

where

$$
v_{I}=-K_{I} \operatorname{sign}(\hat{I}-I)
$$

thus $\dot{\tilde{I}}=v_{I}+\Phi^{T} \tilde{\theta}$. For $K_{I}$ sufficiently chosen large

$$
K_{I}>\left|\Phi^{T} \tilde{\theta}\right|
$$

and assuming that $\hat{\theta}$ is bounded (the proof will be shown later) then a sliding mode regime occurs on the manifold $\tilde{I}=0$ and $0=v_{I e q}+\Phi^{T} \tilde{\theta}$ then

$$
v_{I e q}=-\Phi^{T} \tilde{\theta}
$$


The following approximation is used (see the work of Utkin [16]):

$$
v_{I e q}=\frac{1}{1+\tau s} v_{I}
$$

where $s$ is the Laplace operator and $\tau \rightarrow 0$ is a positive constant.

Hence

$$
\tilde{\theta}=-\left(\Phi \Phi^{T}\right)^{-1} \Phi v_{I e q}
$$

and finally

$$
\dot{\hat{\theta}}=-K_{\theta} \operatorname{sign}(\tilde{\theta})
$$

Let us choose the gain matrix for the estimator such that:

$$
\operatorname{diag}\left(K_{\theta}\right)_{i}>\mu_{\theta_{i}}
$$

Lemma 2. Consider the observer given by (19) and (20); the estimator given by (23)-(25); satisfying assumption 1-B, (21) and (26); then the estimated parameters $\hat{\theta}$ will converge to their real values $\theta(t)$ in finite time.

Proof. Consider the following Lyapunov candidate function

$$
V=\frac{1}{2} \tilde{\theta}^{T} \tilde{\theta} \triangleq \beta(\tilde{\theta})
$$

Its time derivative is:

$$
\begin{aligned}
\dot{V} & =\tilde{\theta}^{T} \dot{\tilde{\theta}}=-\left|\tilde{\theta}^{T}\right|_{G} \operatorname{diag}\left(K_{\theta}\right)-\tilde{\theta}^{T} \dot{\theta} \\
& \leq-\left|\tilde{\theta}^{T}\right|_{G}\left(\operatorname{diag}\left(K_{\theta}\right)-\mu_{\theta}\right)
\end{aligned}
$$

and due to the inequality (26), $\dot{V}$ is negative-definite for $\tilde{\theta} \neq 0$. Therefore the estimation error $\tilde{\theta}$ will converge to 0 in finite time.

\section{Persistent excitation condition.}

It is not necessary that the regressor $\Phi(t)$ satisfies the persistent excitation condition (17), because the estimates are just used in the calculation of $I_{*}\left(\bar{x}_{1 *}, c_{i}\right)$. The only necessary condition is that the estimators approximate the current $I$ by means of its dynamics: $\dot{\hat{I}}\left(\tilde{I}, \Phi^{T} \hat{\theta}\right) \rightarrow \dot{I}\left(x_{1}, c_{i}\right)$ and it is satisfied if:

$$
\Phi^{T} \hat{\theta} \rightarrow \Phi^{T} \theta
$$

\section{Controller Design.}

In order to accomplish the control objectives, the problem has been divided in two parts:

1) To find the control portion that renders the nominal system (6) globally asymptotically stable (GAS).

2) To find the control portion that compensates the uncertain term $\delta(t, x)$ and gives the whole system (3) GAS.

\section{A. Controlling the nominal system.}

In this part of the analysis it is considered that the $x_{2 *}$ reference is well known, i.e. $\tilde{I}(t)=0$.

According to the idea given in [8], the control is decomposed in two parts: one for the steady-state stage $u_{* n}$ and another for the dynamic stage $e_{u n}$.

The reference system is:

$$
\dot{x}_{*}=f\left(t, x_{*}\right)+G\left(t, x_{*}\right) u_{* n}
$$

Hence, the control component for the steady state is:

$$
u_{* n}=\frac{L \dot{x}_{2 *}+v_{g}(t)}{x_{1 *}}
$$

Consider the tracking errors and the variable control:

$$
\begin{gathered}
e=x-x_{*}, e_{\text {un }}=u_{n}-u_{* n} \\
\dot{e}=\dot{x}-\dot{x}_{*}=\left[\begin{array}{c}
\frac{I\left(x_{1}\right)-I_{*}}{C} \\
0
\end{array}\right]+\left[\begin{array}{c}
-\frac{e_{2} u_{* n}}{C}-\frac{\left(e_{2}+x_{2 *}\right) e_{u n}}{C} \\
\frac{e_{1} u_{* n}}{L}+\frac{\left(e_{1}+x_{1 *}\right) e_{u n}}{L}
\end{array}\right]
\end{gathered}
$$

Let us propose the Lyapunov candidate function

$$
V_{n}=\frac{C}{2} e_{1}^{2}+\frac{L}{2} e_{2}^{2}
$$

Its time derivative is:

$$
\begin{aligned}
\dot{V}_{n}= & C e_{1} \dot{e}_{1}+L e_{2} \dot{e}_{2} \\
= & e_{1}\left(I-I_{*}\right)-e_{1}\left[e_{2} u_{* n}+\left(e_{2}+x_{2 *}\right) e_{u n}\right] \\
& +e_{2}\left[e_{1} u_{* n}+\left(e_{1}+x_{1 *}\right) e_{u n}\right] \\
= & -c_{2} e_{1}\left(e^{c_{3} x_{1}}-e^{c_{3} x_{1 *}}\right)-e_{u n}\left(e_{1} x_{2 *}-e_{2} x_{1 *}\right)
\end{aligned}
$$

Consider the controller for the error dynamics of the nominal system:

$$
e_{u n} \triangleq e_{1} x_{2 *}-e_{2} x_{1 *}
$$

thus

$$
\dot{V}_{n}=-c_{2} e_{1}\left(e^{c_{3} x_{1}}-e^{c_{3} x_{1 *}}\right)-\left(e_{1} x_{2 *}-e_{2} x_{1 *}\right)^{2}
$$

From the fact that the first term of the right side of (32) is $<0 \forall e_{1} \neq 0$, the nominal system is GAS.

\section{B. Controlling the whole system.}

The control part of the nominal system was established in (30) and (31). These equations are functions of $\dot{x}_{2 *}$ and $x_{2 *}$, and these values are not exactly known. Therefore, the control for the whole system is

$$
\begin{aligned}
u & =u_{* n}\left(x_{2 *}\right)+e_{u n}\left(x_{2 *}\right)+\Delta u_{*}+\Delta e_{u} \\
& =u_{* n}+e_{u n}+\delta(t, x)
\end{aligned}
$$

where

$$
\delta(t, x) \triangleq \Delta u_{*}+\Delta e_{u}=\frac{L \Delta \dot{x}_{2 *}}{x_{1 *}}+e_{1} \Delta x_{2 *}
$$

is the uncertain term, and $\Delta \dot{x}_{2 *}, \Delta x_{2 *}$ are the perturbations due to the transients in the observer, i.e. $\tilde{I} \neq 0$.

In order to derive the controller for the whole system the next assumption is needed.

Assumption 2. The uncertain term is bounded and known, i.e.

$$
\|\delta(t, x)\|_{2}<\xi
$$

where $\xi$ is a known positive number.

Since this uncertain term satisfies the matching condition, the Lyapunov redesign technique [17] is used to derive a term $w$ that will compensate it.

$$
u=u_{n}+\delta(t, x)+w
$$

where $u_{n}=u_{* n}+e_{u n}$ 
The closed-loop system becomes

$$
\dot{x}=f(t, x)+G(t, x) u_{n}+G(t, x)[w+\delta(t, x)]
$$

and

$$
\dot{e}=f(t, x)+G(t, x) u_{n}-\dot{x}_{*}+G(t, x)[w+\delta(t, x)]
$$

Consider the Lyapunov candidate function for the whole system:

$$
V=\frac{C}{2} e_{1}^{2}+\frac{L}{2} e_{2}^{2}+\beta
$$

where $\beta$ was defined for each estimator in (15) and (27).

Let us omit the arguments of the functions. The time derivative of $V$ is:

$$
\begin{aligned}
\dot{V} & =\frac{\partial V}{\partial e}\left\{f+G u_{n}-\dot{x}_{*}\right\}+\frac{\partial V}{\partial e}\{G[w+\delta]\}+\dot{\beta} \\
& =\dot{V}_{n}+\dot{\beta}+\frac{\partial V}{\partial e} G w+\frac{\partial V}{\partial e} G \delta
\end{aligned}
$$

We can derive the $w$ term as follows:

$$
\frac{\partial V}{\partial e} G w+\frac{\partial V}{\partial e} G \delta \leq \frac{\partial V}{\partial e} G w+\xi\left\|\frac{\partial V}{\partial e} G\right\|_{2}
$$

and now we can choose:

$$
w \triangleq-\xi \frac{\frac{\partial V}{\partial e} G}{\left\|\frac{\partial V}{\partial e} G\right\|_{2}}=-\xi \frac{-e_{1} x_{2}+e_{2} x_{1}}{\left|-e_{1} x_{2}+e_{2} x_{1}\right|}
$$

Theorem 1. Consider the system given by (3)-(4); the estimator given by (11)-(12)|(19)-(25); the controller given by (30), (31) and (39); satisfying assumptions 1-A|1-B and 2 , then, the whole system is globally asymptotically stable. Proof. Using (39) one can write:

$$
\frac{\partial V}{\partial e} G w+\frac{\partial V}{\partial e} G \delta \leq-\xi\left\|\frac{\partial V}{\partial e} G\right\|_{2}+\xi\left\|\frac{\partial V}{\partial e} G\right\|_{2}=0
$$

then, from (16), (28), (32), (38) and (40), $\dot{V}$ is negative definite and the whole system is GAS with the controller given by $u_{n}+w$.

\section{Simulation RESUlts}

Numerical simulations were made in the Simulink/Matlab platform to verify the performance of the estimators and the controller. Two scenarios were simulated. In the first one, realistic variations were applied to the irradiance signal and a ramp to the temperature signal, for both estimators. Graphs a) and b) in figure 3 show these variations.

The adaptive estimator is not able to track step changes since it was designed for constant parameters, and the second scenario was simulated just for the sliding mode estimator. It consists in step changes in both variables, irradiance and temperature. Its values were changed by $50 \%$. This is one of the worst conditions for MPPT in PVS, and because of this, a standard simulation scenario.

For both simulations, the initial conditions in the plant were zero and in the estimators $1 \times 10^{-5}$.

Figures 3 and 4 show the results for the first scenario.

The graphs c) and d) in figure 3 show the first reference $x_{1 *}$, given by the MPPT algorithm, and the capacitor voltage

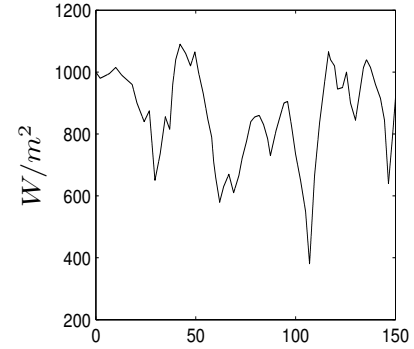

a) Irradiance
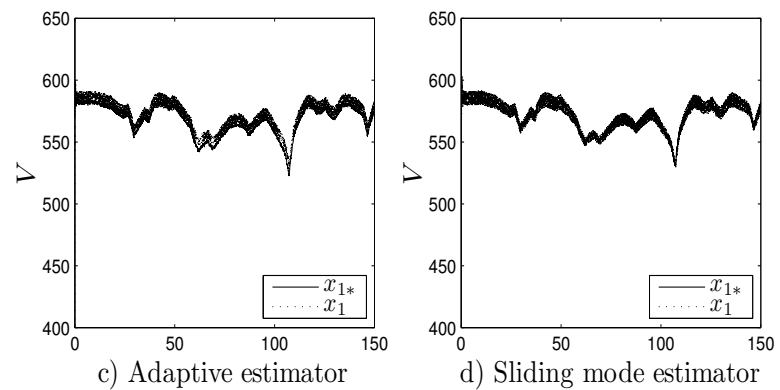

Fig. 3. a) Irradiance signal b) Temperature signal c) $x_{1 *}$ and $x_{1}$ signals in the adaptive estimator d) $x_{1 *}$ and $x_{1}$ signals in the sliding mode estimator

$x_{1}$ for both estimators. The performance of the controller is very good in both cases. The results are pretty similar.

The maximum power point current $I_{*}$ and the maximum power point current estimated $\hat{I}_{*}$ are shown in graphs a) and b) in figure 4 . The adaptive estimator shows bigger deviations from $I_{*}$.

Graphs c) and d) in figure 4 show the output power in both estimators. The results are similar. This is the maximum power that the PVS can deliver with the irradiance and temperature given.

Figure 5 shows the results for the second scenario.

The step changes in irradiance and temperature for the sliding mode estimator are shown in graphs a) and b) in figure 5 . The values were changed by $50 \%$.

The graphs c), d) and e) in the same figure show that the controller and the estimator present a good performance, even under these demanding conditions. Some noticeable ripple appears after the step variations, due not only to the variation speed but also to the variation level (50\%).

The output power is shown in graph e) of the same figure. It clearly follows the environmental variations.

\section{CONCLUding REMARKS AND FUTURE RESEARCH.}

Two solutions for the MPPT of PVS were presented. For the first adaptive scheme control (ASC), an adaptive estimator was designed. It is capable of estimate constant parameters. For the second ASC, a sliding mode estimator was designed. It is capable of estimate time-varying parameters.

A Lyapunov function that proves GAS of the system was derived. The analysis includes the dynamics of the estimators and the uncertainty in the second reference $x_{2 *}$.

Even when GAS was demonstrated for the system including estimators and perturbations, there is one subject 


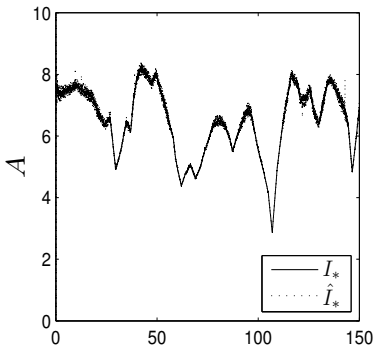

a) Adaptive estimator

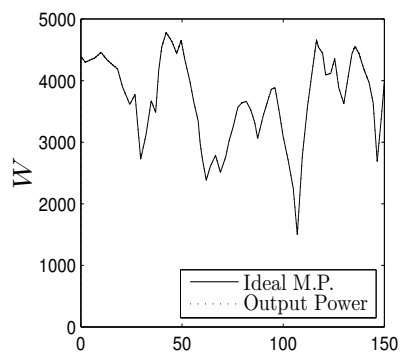

c) Adaptive estimator

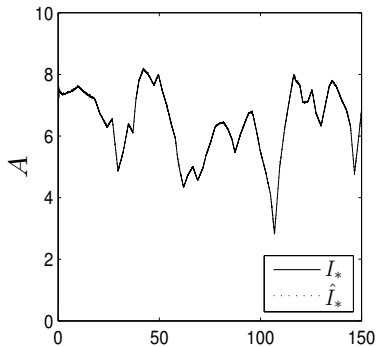

b) Sliding mode estimator

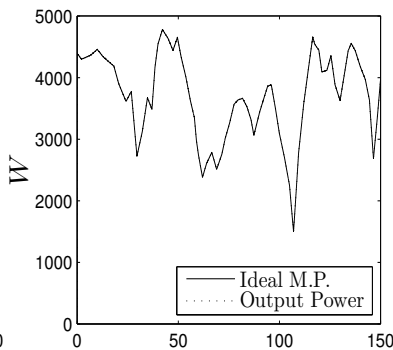

d) Sliding mode estimator
Fig. 4. a) $I_{*}$ and $\hat{I}_{*}$ signals in the adaptive estimator b) $I_{*}$ and $\hat{I}_{*}$ signals in the sliding mode estimator c) Theoretical maximum power and output power in the adaptive estimator case d) Theoretical maximum power and output power in the sliding mode estimator case

that can enrich the analysis, it is the inclusion of the MPPT algorithm in the closed-loop system analysis. We are currently investigating how to achieve this.

Numerical simulations were made to verify the performance of the solutions. Both ASC have good performance under realistic conditions. The sliding mode ASC has the better performance. It can works well even under very demanding conditions.

\section{Acknowledgements}

The work of F. Jaramillo has been supported by CONACyT, Mexico and the French Embassy in Mexico. Also, the authors give thanks to Dr. R. Ortega for his contribution with the adaptive estimator.

\section{REFERENCES}

[1] Soeren Baekhoej Kjaer, John K. Pedersen and Frede Blaabjerg, "A Review of Single-Phase Grid-Connected Inverters for Photovoltaic Modules", IEEE Transactions on Industry Applications, Vol. 41, No. 5, pp. 1292-1306, September-October 2005,

[2] Yaosuo Xue, Liuchen Chang, Soeren Baekhoej Kjaer, Josep Bordonau and Toshihisa Shimizu, "Topologies of Single-Phase Inverters for Small Distributed Power Generators: An Overview", IEEE Transactions on Power Electronics, Vol. 19, No. 5, pp. 1305-1314, September 2004,

[3] Nicola Femia, Giovanni Petrone, Giovanni Spagnuolo and Massimo Vitelli, "Optimization of Perturb and Observe Maximum Power Point Tracking Method", IEEE Transactions on Power Electronics, Vol. 20, No. 4, pp. 963-973, July 2005,

[4] T. Esram and P. L. Chapman, "Comparison of photovoltaic array maximum power point tracking techniques", IEEE Transactions on Energy Conversion, Vol. 22, No. 2, pp. 439-449, June 2007,

[5] Daniel Nahum Zmood and Donald Grahame Holmes, "Stationary Frame Current Regulation of PWM Inverters With Zero Steady-State Error", IEEE Transactions on Power Electronics, Vol. 18, No. 3, pp. 814-822, May 2003,

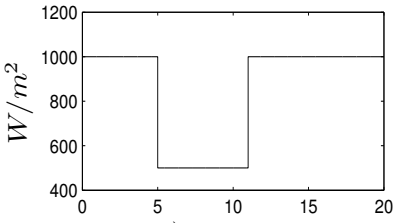

a) Irradiance

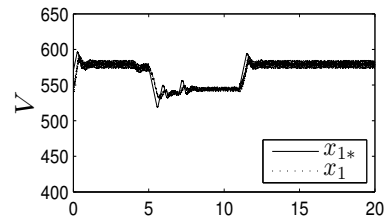

c) Capacitor voltage $x_{1}$

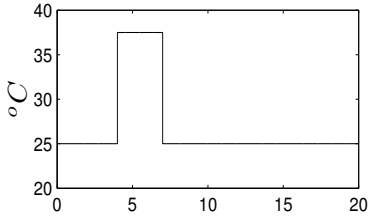

b) Temperature

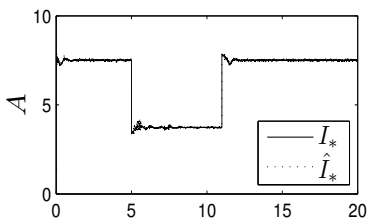

d) MPP current $I_{*}$

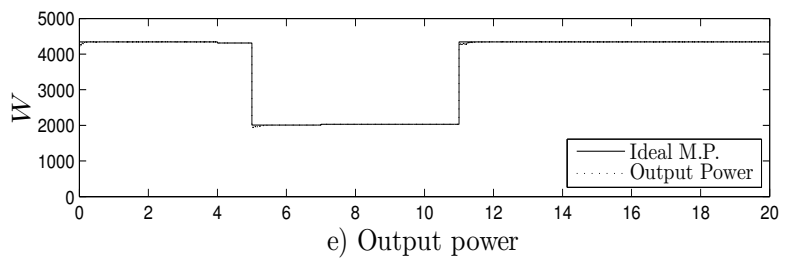

Fig. 5. Step changes and results got in the numerical simulation for the sliding mode estimator. a) Irradiance signal b) Temperature signal c) $x_{1 *}$ and $x_{1}$ signals d) $I_{*}$ and $\hat{I}_{*}$ signals e) Theoretical maximum power and output power

[6] Adrian V. Timbus, Mihai Ciobotaru, Remus Teodorescu and Frede Blaabjerg, "Adaptive Resonant Controller for Grid-Connected Converters in Distributed Power Generation Systems", in Proceedings IEEE Applied Power Electronics Conference 2006, pp. 1601-1606

[7] C. Meza, D. Jeltsema, J. Scherpen, D. Biel, "Passive P-Control of a Grid-Connected Photovoltaic Inverter", in Proceedings of the 17th World Congress, The International Federation of Automatic Control, Seoul, Korea, pp. 5575-5580, July 6-11, 2008

[8] S. Sanders and G. Verghese, "Lyapunov-based control for switched power converters", IEEE Transactions on Power Electronics, Vol. 7, No. 1, pp. 17-24, January 1992,

[9] C. Meza, D. Biel, D. Jeltsema and J.M.A. Scherpen, "LyapunovBased Control Scheme for Single-Phase Grid-Connected PV Centra Inverters", IEEE Transactions on Control Systems Technology, Vol. 20, No. 2, pp. 520-529, March 2012,

[10] J. A. Gow, C. D. Manning, "Development of a photovoltaic array model for use in power-electronics simulation studies", IEEE Proceedings on Electric Power Applications, Vol. 146, No. 2, pp. 193-200, March 1999,

[11] Etienne Saloux, Alberto Teyssedou, Mikhal Sorin, "Explicit model of photovoltaic panels to determine voltages and currents at the maximum power point", Solar Energy, 85, pp. 713-722, 2011,

[12] Thomas Bennett, Ali Zilouchian, Roger Messenger, "Photovoltaic model and converter topology considerations for MPPT purposes", Solar Energy, 86, pp. 2029-2040, 2012,

[13] Jean-Jacques E. Slotine, Weiping Li, Applied Nonlinear Control, Prentice-Hall, 1991

[14] Kumpati S. Narendra, Anuradha M. Annaswamy, Stable Adaptive Systems, Prentice-Hall, 1989.

[15] Godpromesse Kenne, Tarek Ahmed-Ali, F. Lamnabhi-Lagarrigue and Amir Arzande, "Nonlinear systems time-varying parameter estimation: Application to induction motors", Electric Power Systems Research 78 (2008) pp. 1881-1888.

[16] V.I. Utkin, Sliding Modes in Optimization and Control, Springer Verlag, 1992.

[17] H. K. Khalil, Nonlinear Systems 2nd ed., Prentice-Hall, 1996. 